\title{
Endoscopic Transsphenoidal approach for extrasellar extended Pituitary Adenomas: Surgical outcome.
}

\author{
Abdelfattah A. Shehab ${ }^{1}$ MD, Mohammad Fathy Eissa ${ }^{1, *}$ MD, Rabab Yahia Abdelkareem ${ }^{1}$ MB Bch, \\ Tawfik Abdelaaty Elkholy ${ }^{2}$ MD.
}

*Corresponding Author:

Mohammad FathyEissa

mohammadfathyeissa@azhar.edu.eg

Received for publication January 15,

2020; Accepted March 8, 2020;

Published on line March 11, 2020.

Copyright 2020 The Authors published by Al-Azhar University, Faculty of Medicine, Cairo, Egypt. All rights reserved. This an open-access article distributed under the legal terms, where it is permissible to download and share the work provided it is properly cited.

The work cannot be changed in anyway or used commercially.

doi: 10.21608/aimj.2020.22458.1081

${ }^{I}$ Neuro Surgery Department, Faculty of Medicine for Girls, Al-Azhar University, Cairo, Egypt

${ }^{2}$ Otorhinolaryngology Department,

Faculty of Medicine for Girls, Al-Azhar

University, Cairo, Egypt

\begin{abstract}
Background: Transsphenoidal approaches usually are the preferred method for treatment of pituitary tumors because of a lower risk of complications. Recently, endoscope has been introduced as an alternative and effective tool in transsphenoidal surgery. This study was conducted at Al-Azhar University Hospitals and Nasr City Health Insurance Hospital to evaluate the role of endoscope in dealing with pituitary tumors with suprasellar and parasellar extensions.

Patients and methods: A total of 32 cases with pituitary adenoma with extrasellar extension were included. All cases were clinically and radiologically assessed. Pituitary hormones were also assessed. The endoscopic transsphenoidal endonasal procedure was performed for all included cases by the same surgical team.

Results: The mean age of the included cases was 41.35 years. The mean size of pituitary lesions was $39.15 \mathrm{~mm}$. Total resection was achieved in 22 cases $(68.8 \%)$ while subtotal resection was achieved in 10 cases $(31.2 \%)$. Complications included vascular injury $(6.25 \%)$, visual deterioration $(9.4 \%)$, deterioration of consciousness $(9.4 \%)$, transient diabetes insipidus (6.25\%), hydrocephalus $(6.25 \%)$ and sinonasal symptoms $(15.6 \%)$. There was a significant improvement of visual symptoms after surgery. Follow up showed a decrease in the number of cases having endocrinopathy although it was not significant ( $p$ $=0.052$ )

Conclusion: Endoscopic transsphenoidal surgery is the preferred method for removal of the pituitary tumors because it provides better visualization and makes the orientation in the operative field easier and safer than the microscopic transsphenoidal approach. Also, we recommend to work in combination with E.N.T. to achieve the best results.
\end{abstract}

Keywords: Endoscopic transsphenoidal; Extrasellar extension; Pituitary ; Macroadenoma.

Disclosure: The authors have no financial interest to declare in relation to the content of this article. The Article Processing Charge was paid for by the authors.

Authorship: All authors have a substantial contributions to the article

\section{INTROUDTION}

The pituitary gland composed of anterior and posterior lobes. The gland secretes eight peptide hormones, two from the posterior lobe and six from the anterior lobe. Pituitary adenomas are by far the most common tumours of the sellar region, comprising 90 to $95 \%$ of all such tumours ${ }^{1,3}$. Clinically, pituitary tumors usually present either due to endocrinologic disturbance, and/or due to mass effect. Pituitary macroadenoma may produce headache. A small number present with pituitary apoplexy². In the last 10 years the endoscopic endonasal transsphenoidal approach has been proposed as a minimally invasive procedure for the treatment of pathologies of the sellar region ${ }^{4}$. This procedure is performed via a wide anterior sphenoidotomy with detachment of the septum from the sphenoid face, and avoids the use of a transsphenoidal retractor and any intraoral or nasal incisions. Straight and angled endoscopes are used throughout the procedure to provide a wide view of the sella and are manipulated by a co-surgeon. 5,6

\section{PATIENTS AND METHODS}

Study design: This is a prospective study that was conducted in Al-Azhar University Hospitals and Nasr City Health Insurance Hospital during the period between January 2016 and July 2019.

A total of 32 cases with pituitary macroadenomas with extrasellar extension that had undergone total or subtotal resection performed by endoscopic endonasal transsphenoidal surgery were included in the study. All cases were subjected to complete history taking, thorough neurological examination including visual examination. In addition, routine preoperative laboratory investigations including pituitary hormonal profile was performed. CT of PNS was done for all cases to determine the precise anatomic features of the surgical route. Moreover, tumor size was evaluated by high resolution MR imaging for all cases.

\section{Operative procedure:}

The patient was under general anesthesia with endotracheal intubation, in a supine position with the trunk elevated $10^{\circ}$ and the head turned $10^{\circ}$ toward the surgeon. 
Abdelfattah et al, Transsphenoidal Approach

A rigid $0^{\circ}$ endoscope, $25 \mathrm{~cm}$ in length, $4 \mathrm{~mm}$ in diameter was introduced through the nostril. A cottonoids soaked with diluted adrenaline (1:100000) were positioned between the middle turbinate and the nasal septum in each nostril to enlarge the space between them and obtain decongestion of the nasal mucosa.

After the creation of adequate space between the middle turbinate and the nasal septum, the endoscope was directed upward along the roof of the choana and the sphenoethmoid recess until it reached the sphenoid ostium. If the sphenoid ostium was not visible and the choana was identified, the endoscope was advanced along the sphenoethmoid recess for approximately 1.5 $\mathrm{cm}$ above the upper edge of choana. Lateral dissection of mucosa covering the most posterior $1 \mathrm{~cm}$ of nasal septum and also sphenoid face between two ostia, Anterior sphenoidotomy with removal of approximately $1 \mathrm{~cm}$ of the posterior part of the nasal septum were done. After the sphenoidotomy, removal of the sphenoid septa was performed then the posterior and lateral walls of the sphenoid sinus were visible, with the sellar floor at the center. The opening of the sellar floor was performed and must be extended as required by the specific pathological entity.

The dura was incised in a midline position and in a linear or cruciate fashion. The removal of macroadenomas was accomplished sequentially. The inferior and lateral fragments of the lesion were removed before the superior aspect. If there was enough space in the sellar cavity and angled 30-degree endoscope were then advanced sequentially into the tumor cavity to verify the presence of any tumor remnants, which often were in the recesses created by the fall of the suprasellar cistern.

When the lesion extended outside the sella, its removal was accomplished, under endoscopic control, by use of curved suction cannulas. At the end of the procedure, haemostasis was done and final irrigation of the nasal cavities was performed; then, the endoscope was removed gradually, and the middle turbinate was gently put back in a medial direction. Packing of the nasal cavity was used to be removed after 48 hours, except in case of intra-operative CSF leak nasoseptal Flap was done and the nose was packed for 3-5 days.

Uncomplicated cases stayed in the hospital for 3 days, nasal packs were removed on the second postoperative day. The patient was monitored for nasal or post nasal CSF drainage for at least several hours after removal of the packs. Patients with pituitary adenoma rather than ACTH secreting adenoma were given hydrocortisone $100 \mathrm{mg}$ every 8 hours and tapered on the next 5 days.The patient neurological condition, vital signs, CSF leakage, urine output, hormonal profile, laboratory investigation, pathological diagnosis of the tumor and the patient medication at home were reviewed, and recorded in the hospital records.

All of the 32 patients were followed up postoperatively for a period ranging from 6 months to 28 months (mean 12 months) clinically, endocrinologically and radiologically.

\section{Case Presentation:}

Male patient, 29 years old presented by severe agonizing headache with visual deterioration and impotence and his images in the following figure (1)
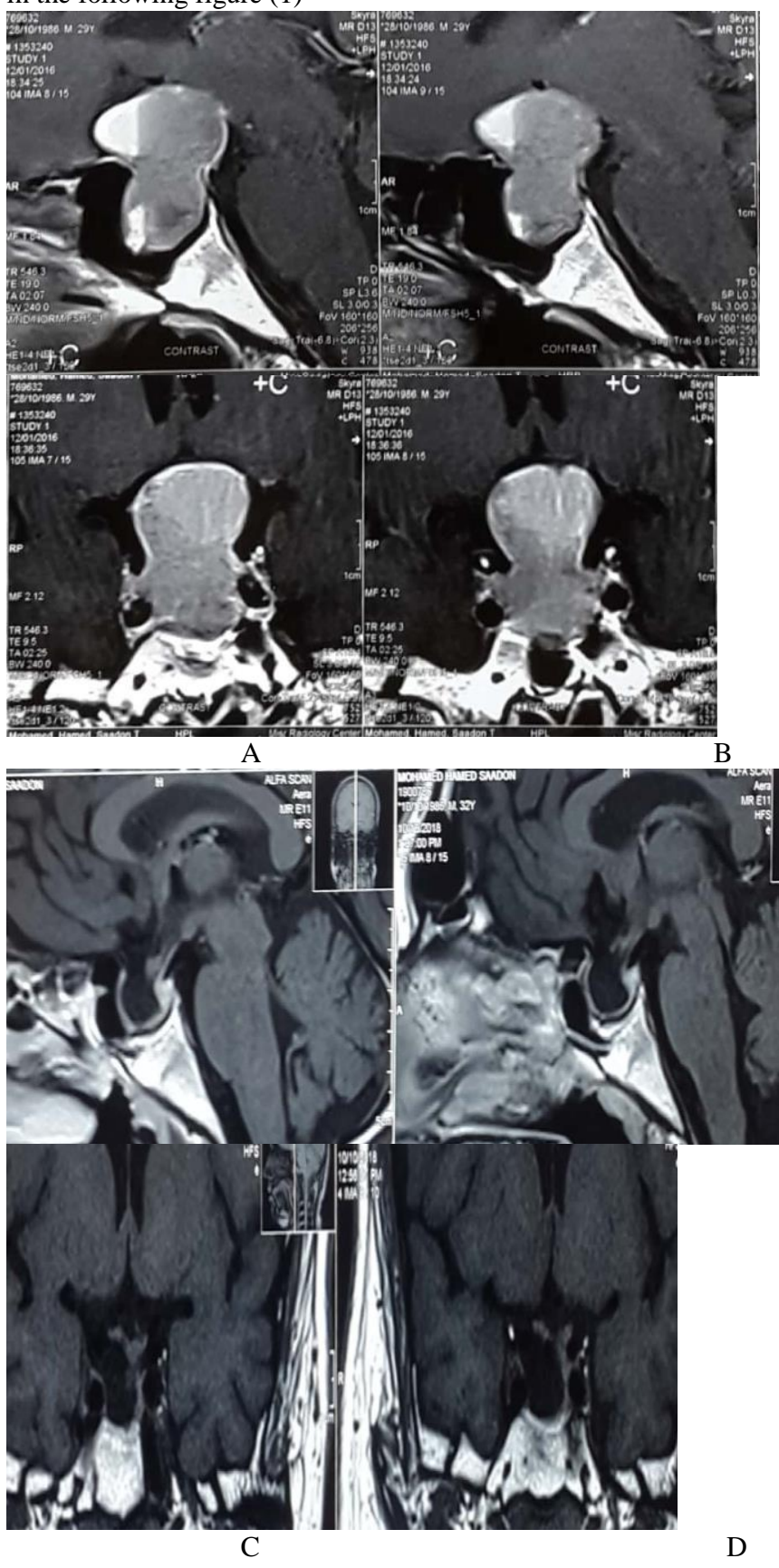

Fig: 1 shows the following:

A. Sagittal view MRI T1 with contrast of Sella showing huge sellar and suprasellar mass widening sella turcica and compressing optic apparatus above with areas of hemorrhage inside.

B. Coronal view MRI T1 with contrast of the same patient

C. Postoperative Sagittal view MRI sella with contrast of the same patient showing complete disappearance of the previously noted Sellar and Suprasellar Pituitary adenoma with apoplexy which proved by histopathological examination.

D. Postoperative Coronal view MRI T1 with contrast of the same patient. 
Data were collected, revised, coded and entered to the Statistical Package for Social Science (IBM SPSS) version 20. Qualitative data were presented as number and percentages while quantitative data were presented as mean, standard deviations and ranges.

The comparison between two groups with qualitative data were done by using Chi-square test. The comparison between two independent groups with quantitative data and parametric distribution were done by using Independent t-test. P value < 0.05 was considered to be significant.

\section{RESULTS}

The mean age of the included cases was 41.35 years (range, $24-$ 55). We included 19 females (59.4\%) and 13 males (40.6\%). Non-secretory adenoma was the commonest type encountered in our study (14 cases $-43.8 \%$ ), followed by prolactinoma (10 cases $-31.2 \%)$. The remaining 8 cases $(25 \%)$ had growth hormone secreting adenoma. The mean size of the tumours in our study was $39.15 \mathrm{~mm}$ (range, $30-46$ ). These data are illustrated at table 1.

\begin{tabular}{|l|l|c|}
\hline \multirow{2}{*}{ Variable } & No $=32$ \\
\hline \multirow{2}{*}{ Age (years) } & Mean + SD & $41.35 \pm 9.26$ \\
& Range & $24-55$ \\
\hline \multirow{2}{*}{ Sex } & Female & $19(59.4 \%)$ \\
& Male & $13(40.6 \%)$ \\
\hline \multirow{3}{*}{ Type } & GH & $8(25 \%)$ \\
& N secretory & $14(43.8 \%)$ \\
& PRL & $10(31.2 \%)$ \\
\hline \multirow{2}{*}{ Size $(\mathrm{mm})$} & Mean + SD & $39.15 \pm 5.40$ \\
& Range & $39.15 \pm 5.40$ \\
\hline
\end{tabular}

Table 1: Patient and tumour characteristics.

Regarding clinical presentation, headache was the commonest complaint encountered ( 26 cases $-81.25 \%$ ), followed by visual affection (22 cases - 68.75\%). Hormonal disturbances were present in 19 cases (59.4\%) whereas hormonal therapy was reported by 10 cases $(31.25 \%)$. These data are illustrated at table 2 below.

\begin{tabular}{|l|c|}
\hline \multicolumn{1}{|c|}{ Complaint } & No (\%) \\
\hline Headache & $26(81.25 \%)$ \\
\hline Visual affection & $22(68.75 \%)$ \\
-Diminution of vision & $-22(68.75 \%)$ \\
-Field affection & $-22(68.75 \%)$ \\
-Papilledema & $-3(9.4 \%)$ \\
\hline Hormonal disturbances & $19(59.4 \%)$ \\
-Acromegaly & $-8(25 \%)$ \\
-Amenorrhea and galactorrhea & $-8(25 \%)$ \\
-Loss of libido & $-2(6.25 \%)$ \\
-Pan hypopituitarism & $-1(3.15 \%)$ \\
\hline Hormonal replacement therapy & $10(31.25 \%)$ \\
\hline Table (2): Clinical presentations.
\end{tabular}

Regarding to tumour extension, suprasellar extension was present in 21 cases $(65.6 \%)$. Five cases had suprasellar while bilateral parasellar extension was present in 8 cases $(25 \%)$. The remaining cases had suprasellar as well as unilateral parasellar extension $(9.4 \%)$. These data are shown at table 3 .

\begin{tabular}{|c|c|}
\hline Tumour extension & No $(\%)$ \\
\hline Suprasellar & $21(65.6 \%)$ \\
\hline Para + Suprasellar bilateral & $8(25 \%)$ \\
\hline Para + Suprasellar unilateral & $3(9.4 \%)$ \\
\hline
\end{tabular}

Table 3: Tumour extension.

Vascular injury occurred in 2 cases (6.25\%). Deterioration of visual symptoms was reported in 3 cases (9.4\%) while deterioration of consciousness was encountered in 3 cases (9.4\%). Diabetes insipidus occurred in 2 cases (6.25\%). Furthermore, CSF leak was encountered in 2 cases $(6.25 \%)$ whereas hydrocephalus was present in 2 cases $(6.25 \%)$. Sinonasal symptoms were experienced in 5 cases (15.6\%). Mortality was not encountered in our study ( $0 \%)$. Table 4 illustrates these data.

\begin{tabular}{|l|c|}
\hline \multicolumn{1}{|c|}{ Variable } & No $(\%)$ \\
\hline Vascular injury & $2(6.25 \%)$ \\
\hline Visual deterioration & $3(9.4 \%)$ \\
\hline Deterioration of consciousness & $3(9.4 \%)$ \\
\hline Transient diabetes insipidus & $2(6.25 \%)$ \\
\hline Hydrocephalus & $2(6.25 \%)$ \\
\hline Sinonasal symptoms & $5(15.6 \%)$ \\
\hline
\end{tabular}

Table 4: Intra- and post-operative complications. 
When comparing visual symptoms after operation with the preoperative findings, it was evident that surgery significantly Improve the visual manifestations. Although papilledema resolved in the three cases, it was not statistically significant change $(p=0.146)$. These data are illustrated at table 6 .

\begin{tabular}{|c|c|c|c|}
\hline & $\begin{array}{c}\text { Preoperati } \\
\text { ve }\end{array}$ & $\begin{array}{c}\text { Post- } \\
\text { operative }\end{array}$ & $\begin{array}{c}P \\
\text { value }\end{array}$ \\
\hline $\begin{array}{c}\text { Diminution of } \\
\text { vision }\end{array}$ & $\begin{array}{c}22 \\
(68.75 \%)\end{array}$ & $3(9.4 \%)$ & 0.00 \\
& & 1 \\
\hline Field of & 22 & $3(9.4 \%)$ & 0.00 \\
vision & $(68.75 \%)$ & & 1 \\
\hline Papilledema & $3(9.4 \%)$ & $0(0 \%)$ & 0.14 \\
& & & 6 \\
\hline
\end{tabular}

Table 5: Comparison between preoperative and

postoperative visual assessment.

As illustrated in table 6, cases with hormonal disturbances has decreased down to 17 (53.1\%) at 6-month follow up, and 8 cases (25\%) at 12-month follow up. Nevertheless, it was not considered statistically significant $(\mathrm{p}=0.052)$.

\begin{tabular}{|l|c|c|c|}
\hline & $\begin{array}{c}6 \\
\text { months }\end{array}$ & $\begin{array}{c}12 \\
\text { months }\end{array}$ & $\begin{array}{c}P \\
\text { value }\end{array}$ \\
\hline $\begin{array}{l}\text { Hormonal } \\
\text { disturbances }\end{array}$ & $\begin{array}{c}17 \\
(53.1 \%)\end{array}$ & $\begin{array}{c}8 \\
(25 \%)\end{array}$ & 0.052 \\
\hline
\end{tabular}

Table 6 : Hormonal assessment at follow up.

On post-operative radiological assessment, total tumour extension was achieved in 22 cases $(68.75 \%)$ while the remaining 10 cases had subtotal excision $(31.25 \%)$. Tumours with smaller diameter were more amenable to total resection, whereas subtotal resection was achieved in larger tumours $(p=0.025)$. These data are illustrated at table (7).

\begin{tabular}{|c|c|c|c|}
\hline & $\begin{array}{c}\text { Total resection } \\
(n=22)\end{array}$ & $\begin{array}{c}\text { Subtotal } \\
\text { resection }(n=10)\end{array}$ & $\begin{array}{c}P \\
\text { value }\end{array}$ \\
\hline $\begin{array}{c}\text { Mean } \\
\pm \mathrm{SD}\end{array}$ & $37.43 \pm 5.37$ & $43.17 \pm 2.86$ & 0.0 \\
Range & $30-44$ & $38-46$ & 25 \\
\hline
\end{tabular}

Table 7: Post-operative radiological assessment

\section{DISCUSSION}

This study included 32 patients with pituitary macroadenomas with extrasellar extension (defined as maximum diameter $\geq 3$ $\mathrm{cm}$ ) operated by endoscopic transsphenoidal approach from Jan 2016 to July 2019.

In this study, pituitary tumors were more frequent in females $(59.4 \%)$ and the age of the patients ranged from $24-55$ years (mean age of $41.35 \pm 9.26$ years) with extrasellar extension (defined as maximum diameter $\geq 3 \mathrm{~cm}$ ).

Other endoscopic endonasal resection studies, performed by Chabot et al., was performed on 39 patients whose age ranged from 23 - 80 years with predominant male $(64.1 \%)$ to female $(38.8 \%)$ with large $(>3 \mathrm{~cm})$ or giant $(>4 \mathrm{~cm})$ pituitary macroadenomas pituitary macroadenomas that were surgically treated during the period 2009-2014 at Geisinger Medical Center and North Shore University Hospital ${ }^{7}$.

Juraschka et al. reviewed 487 patients who underwent endoscopic endonasal transsphenoidal resection of sellar masses. From this group, 73 consecutive patients with large and giant pituitary adenomas (defined as maximum diameter $\geq 3 \mathrm{~cm}$ ) who underwent endoscopic endonasal transsphenoidal surgery between January 2006, and June $2012^{8}$.

In the present study, visual symptoms were the first most important presentations because $68.75 \%$ of patients with pituitary adenoma had suprasellar extension with subsequent compression on the optic apparatus. Diminished visual acuity and visual field defects were present in 22 patients, and fundus examination papilloedema in 3 patients $9.4 \%$.

This finding also detected in studies performed by Chabot et al., reported that more than $74.4 \%$ of patients presented with visual field defects and (62.2\%) of decreased visual acuity ${ }^{7}$.

While, Juraschka et al., have 52 patients suffering from visual defect ( $97 \%$ ) and 45 patients of decreased visual acuity $(83 \%)^{8}$.

Headache was the most frequent symptom in this study and it was found in $81.25 \%$ of the patients, severity of headache ranged from mild to severe form. While Chabot et al., reported $46.2 \%$ of patients having headache ${ }^{7}$.

Symptoms and signs of hormonal disturbance were common findings in this study (59.4\%), prolactinomas were in 10 patients $(31.25 \%)$, acromegalic features were in 8 patients $(25 \%)$. Pituitary insufficiency was present in 1 patient $(3.15 \%)$ while non-secretory adenomas presented in 8 patients (40.6\%).

Our results also compatible with Chabot et al., who reported 26 $(66.7 \%)$ of patients with endocrinopathy. There were $16(41 \%)$ patients with hypopituitarism requiring hormone replacement therapy (HRT) before surgical treatment. Of these patients, 2 had adrenocorticotropic hormone secreting tumors (Cushing syndrome), 1 had a GH-secreting tumor, and 3 had prolactinoma ${ }^{7}$.

Komotar et al., also founded that, excessive secretion of growth hormone was present in $6 / 106$ cases $(5.7 \%)$, prolactin in $10 / 106$ cases $(9.4 \%)$, ACTH in $1 / 106$ cases $(0.9 \%)$, and FSH in $6 / 106$ cases $(5.7 \%)^{9}$.

In our study, pituitary adenomas with suprasellar extension were found in 21 patients (65.6\%) while suprasellar extension with cavernous sinus invasion were found in 11 cases $(34.4 \%), 3$ of them $(9.4 \%)$ with unilateral extension \& 8 of them $(25 \%)$ with bilateral extension with no compatibility. 
As regards patient's outcome; $90 \%$ of the patients with visual field defects improved postoperatively, $90 \%$ of the patients with diminished visual acuity improved after surgery and fundus improved. The basis for these favorable endoscopic results as regards the visual improvement were attributed to better visualization of suprasellar area.

In another study, all 38 patients had a good visual outcome. No patients had new postoperative visual acuity or visual field deficits. Patients with bilateral visual symptoms were more likely to show improvement in postoperative visual fields and visual acuity testing. There was no statistically significant association between maximum diameter and visual outcomes, and there was no correlation between the extent of resection and improvement in visual symptoms?

Another study reported that $73 \%$ of patients experienced improvement in visual acuity, while $24 \%$ were unchanged. Visual fields were improved in $61.8 \%$ and unchanged in $5.5 \%$. Compared to the open cohort, the endoscopic cohort had higher rate of improved visual outcome (91.1\% vs. $45.7 \%$; P\0.003). The microscopic transsphenoidal cohort had higher rate of worse visual outcomes $(34.8 \%)$ than the endoscopic group $(91.1 \%)^{9}$.

In our study the outcome of postoperative hormonal assessment was done for follow up of our patients at 6 and 12 months. The prolactin hormone level normalized in 2 out of $6(33.3 \%)$ patients through the first 6 months. At the end of 12th month of follow up, improvement of another 2 patients was obtained with total 4 out of $6(66.7 \%)$. While, prolactin hormone level did not return to its normal value and remained high $200-400 \mathrm{ng} / \mathrm{ml}$ in only one patient and received bromocriptine postoperatively for 5 months until the hormone returned to its normal values. The remaining patient with persistently high PRL level continued the dopamineagonist therapy after surgery.

Growth hormone level normalized in 4 out of $5(80 \%)$ after 6 months then become 2 out of $5(40 \%)$ at the end of 12 months. With continuous follow up one of patients returns to normal value after one and half year from the surgery and one patient still unimproved due to subtotal resection.

In our study one patient submitted to radiosurgery (gamma knife) due to residual part of the tumor with improvement of the tumor size. Chabot and his colleagues reported that 34 patients had a good endocrine outcome, whereas 5 did not. Also, 5 patients with preoperative endocrinopathy were able to discontinue their medical therapy. A preoperative endocrinopathy was present in 26 patients, with 16 requiring HRT before surgery.
Only 1 patient without preexisting endocrinopathy developed new abnormalities requiring treatment. Endocrine function was preserved in the other 3 patients whereas the other 2 had improved but elevated levels of prolactin and were kept on their preoperative dosage of bromocriptine or cabergoline. The patient with a GH-secreting tumor underwent GTR and chemical remission of his disease ${ }^{7}$.

As regards the radiological outcome in our study, follow up MRI revealed complete tumor removal in 14 patients $70 \%$ Subtotal tumor resection in 6 patients 30\%. Also, patients presented with suprasellar extension achieve more resection 9 out of 12 patients $(75 \%)$ rather than those with cavernous extension 4 out of 8 patients (50\%). The incomplete removal of the tumor was due to the fibrous nature of the tumor and the presence of part of the tumor above and laterals to the carotid artery or encasing it.

Extent of resection were reviewed by Chabot and his associates, and it was classified into 1 of 3 categories: 22 (56.4\%) patients had GTR (gross total resection), 11 (28.2\%) had NTR (near total resection), and $6(15.4 \%)$ had STR (subtotal resection) ${ }^{7}$.

In another study, gross-total resection was achieved in 16 patients (24\%), near-total in $11(17 \%)$, subtotal in $24(36 \%)$, and partial in $15(23 \%)^{8}$.

In the present study CSF leakage was the commonest intraoperative problems found in 2 patients out of 20 (10\%). For both of them packing sella in layers was done with or without glue. After surgery one of them continued to have leakage in the early post-operative period that required bed rest and conservative management with improvement.

Chabot et al., reported CSF leaks in $10.3 \%$. There were 21 documented intraoperative CSF leaks. With the routine use of a nasoseptal flap, only 4 patients developed postoperative CSF leaks. Of these, 3 patients had undergone surgery for recurrent tumor and required repair with a fat graft. None of these 3 patients had a viable nasoseptal flap because of prior surgery or radiation. The fourth patient had a CSF leak from the ethmoidal recess, which was discovered postoperatively when the patient developed meningitis and pneumocephalus. This last patient required repeat surgery for repair of CSF leak in a delayed fashion. The other 3 patients were managed successfully with lumbar drainage. There were no permanent complications from postoperative CSF leak in any of the 4 patients ${ }^{7}$. 
In the present study 2 patients $(6.25 \%)$ had post-operative diabetes insipidus (DI) in the early postoperative period and It was a transient for no need for replacement therapy.

Komotar and his associates reported that postoperative complications included permanent DI in $4.7 \%$ in endoscopic endonasal (5/106) and $8.7 \%$ in transsphenoidal microscopic approach $^{9}$.

\section{CONCLUSION}

According to the previous results, endoscopic transsphenoidal surgery is the preferred method for removal of the pituitary tumors. It provides better visualization and makes the orientation in the operative field easier and safer for removal of extended extrasellar pituitary macroadenomas. Also, study recommends multidisciplinary approach with E.N.T. specialty to achieve the best results.

\section{REFERENCES}

1. Mehta GU, Lonser RR. Management of hormone-secreting pituitary adenomas. Neuro-oncology. 2017;19(6):762-73.

2. Chanson P, Raverot G, Castinetti F, et al. Management of clinically non-functioning pituitary adenoma. Annales d'endocrinologie. Ann Endocrinol (Paris). 2015; 76(3):239-47. doi: 10.1016/j.ando.2015.04.002

3.Li A, Liu W, Cao P, et al T. Endoscopic versus microscopic transsphenoidal surgery in the treatment of pituitary adenoma: a systematic review and meta-analysis. World neurosurgery. 2017; 101:236-46.

4. Gondim J, Almeida J, de Albuquerque L, et al. Endoscopic endonasal transsphenoidal surgery in elderly patients with pituitary adenomas. Journal of neurosurgery. 2015;123 (1):31-8.

5. Buchfelder M, Zhao Y, Schlaffer S. Advances in pituitary surgery. Current Opinion in Endocrine and Metabolic Research. 2018; 1:51-6.

6. Rosen M, Saigal, Evans Jet al. A review of the endoscopic approach to the pituitary through the sphenoid sinus. Current opinion in otolaryngology \& head and neck surgery. 2006;14 (1):6-13
7.Chabot D, Chakraborty S, Imbarrato G, et al. Evaluation of outcomes after endoscopic endonasal surgery for large and giant pituitary macroadenoma: a retrospective review of 39 consecutive patients. World neurosurgery. 2015;84(4):978-88. 8.Juraschka K, Khan OH, Godoy BL, et al. Endoscopic endonasal transsphenoidal approach to large and giant pituitary adenomas: institutional experience and predictors of extent of resection. Journal of neurosurgery. 2014;121 (1):75-83.

9. Komotar J, Starke M, Raper M, et al. Endoscopic endonasal compared with microscopic transsphenoidal and open transcranial resection of giant pituitary adenomas. Pituitary. 2012;15 (2):150-9. 